\title{
Lithium posttreatment confers neuroprotection through glycogen synthase kinase-3 $\beta$ inhibition in intracerebral hemorrhage rats
}

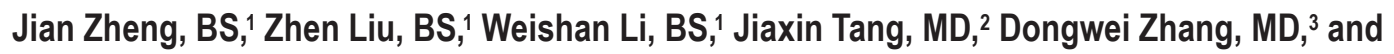 \\ Xiaobo Tang, MD, PhD'
}

\begin{abstract}
'Department of Biopharmaceutical Sciences, College of Pharmacy, Harbin Medical University, Heilongjiang; ${ }^{2}$ The Ninth People's Hospital, School of Medicine, Shanghai Jiaotong University, Shanghai; and ${ }^{3}$ Department of General Surgery, The Second Affiliated Hospital of Harbin Medical University, Heilongjiang, China
\end{abstract}

\begin{abstract}
OBJECTIVE Inflammation and apoptosis are two key factors contributing to secondary brain injury after intracerebral hemorrhage $(\mathrm{ICH})$. The objective of this study was to evaluate the effects of lithium posttreatment on behavior, brain atrophy, inflammation, and perihematomal cell death. Furthermore, the authors aimed to determine the role of the proapoptotic glycogen synthase kinase-3 $\beta$ (GSK-3 $\beta$ ) after experimental ICH.

METHODS Male Sprague-Dawley rats $(n=108)$ were subjected to intracerebral infusion of semicoagulated autologous blood. Window of opportunity and dose optimization studies of lithium on $\mathrm{ICH}$-induced injury were performed by measuring neurological deficits. Animals with $\mathrm{ICH}$ received vehicle administration or lithium posttreatment $(60 \mathrm{mg} / \mathrm{kg})$ for up to 21 days. Hemispheric atrophy was evaluated. Perihematomal cell death was quantified through terminal deoxynucleotidyl transferase-mediated deoxyuridine triphosphate nick-end labeling (TUNEL). The number of myeloperoxidase (MPO)-positive neutrophils and OX42-positive microglia in the perihematomal areas were calculated. Western blotting

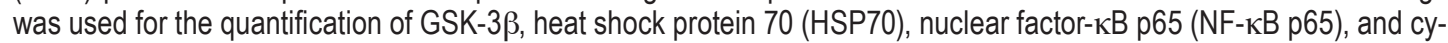
clooxygenase-2 (COX-2).
\end{abstract}

RESULTS Lithium, at a dose of $60 \mathrm{mg} / \mathrm{kg}$ initiated from 2 hours after injury, exhibited the best effects of improving neurological outcomes $3,5,7,14,21$, and 28 days after $\mathrm{ICH}$, reduced the hemispheric atrophy at 42 days after surgery, and reduced the number of TUNEL-positive cells, MPO-positive neutrophils, and OX42-positive microglia in the perihematomal areas. Furthermore, lithium posttreatment modulated GSK-3 $\beta$, increased HSP70, and decreased NF- $\mathrm{KB}$ p65 and COX-2 expression in the ipsilateral hemisphere.

CONCLUSIONS Lithium posttreatment at a dose of $60 \mathrm{mg} / \mathrm{kg}$, initiated beginning 2 hours after injury, improves functional and morphological outcomes, and inhibits inflammation and perihematomal cell death in a rat model of semicoagulated autologous blood ICH through inactivation of GSK-3 $\beta$.

https://thejns.org/doi/abs/10.3171/2016.7.JNS152995

KEY WORDS intracerebral hemorrhage; inflammation; apoptosis; lithium; glycogen synthase kinase-3 $\beta$; neurological deficit; vascular disorders

I NTRACEREBRAL hemorrhage (ICH) represents 10\%-15\% of all cerebrovascular events, and is associated with considerable disability and a high mortality rate. ${ }^{21} \mathrm{Al}-$ though the pathophysiological mechanisms of ICH are still unclear, inflammation and apoptosis are two key factors contributing to secondary brain damage, suggesting that antiinflammatory approaches and inhibition of apoptosis may lessen the consequences of hemorrhagic stroke. ${ }^{1,14,27}$
Lithium has strong antiinflammatory and antiapoptotic effects. ${ }^{4,24}$ Pretreatment of ICH rats with lithium, via antiinflammation, reduced perihematomal cell death, which was associated with sensorimotor recovery..$^{15}$ However, pretreatment of patients with ICH using lithium is almost impossible before onset of the disease, which means pretreatment has little practical clinical meaning. Intending to achieve a translational perspective, as well as to probe the

ABBREVIATIONS COX-2 = cyclooxygenase-2; CRMP2 = collapsin response mediator protein 2; GSK-3 $\beta$ = glycogen synthase kinase-3 $\beta$; HSF-1 = heat-shock factor-1; HSP70 = heat shock protein 70; ICH = intracerebral hemorrhage; $\mathrm{MPO}=$ myeloperoxidase; $\mathrm{NF}-\kappa \mathrm{B}=$ nuclear factor- $\mathrm{\kappa B} ; \mathrm{p}-\beta$-catenin $=$ phosphorylated- $\beta$-catenin; $p$-CRMP2 $=$ phosphorylated-CRMP2; p-GSK-3 $\beta=$ phosphorylated-GSK-3 $\beta$; PBS = phosphate-buffered saline; TBST = Tris-buffered saline with Tween; TUNEL = terminal deoxynucleotidyl transferase-mediated deoxyuridine triphosphate nick-end labeling.

SUBMITTED December 23, 2015. ACCEPTED July 13, 2016.

INCLUDE WHEN CITING Published online October 14, 2016; DOI: 10.3171/2016.7.JNS152995. 
long-term effects of lithium in addition to the short-term effects on ICH, we designed the current study in which rats with ICH were posttreated with therapeutically relevant concentrations of lithium for 21 days.

Although the mechanisms of lithium therapy are not fully elucidated, pharmacological and genetic studies indicate that inactivation of glycogen synthase kinase- $3 \beta$ (GSK-3 $\beta$ ) is one of the main mechanisms of lithium. ${ }^{4}$ In the present study, we hypothesized that lithium, when administered after the induction of ICH through inhibition of GSK-3 $\beta$, alleviated inflammation and cell apoptosis, and subsequently promoted neurological recovery and ameliorated morphological outcomes (brain atrophy) in a rat model of $\mathrm{ICH}$.

\section{Methods \\ Induction of $\mathrm{ICH}$}

Animal protocols for these studies were approved by the Experimental Animal Ethics Committee of Harbin Medical University, China. Efforts were made to minimize animal suffering and to reduce the number of animals used. One hundred eight male Sprague-Dawley rats (Experimental Animal Center of the Second Affiliated Hospital, Harbin Medical University, Harbin, China), weighing between 250 and $300 \mathrm{~g}$, were used in the experiments. The rats were kept under environmentally controlled conditions (12 hours light/dark cycle at $20^{\circ}-23^{\circ} \mathrm{C}$ ) and $50 \%$ relative humidity with enough food and water. Experimental ICH was induced by the stereotactic intrastriatal administration of semicoagulated autologous whole blood, as described in our previous study. ${ }^{20}$

Briefly, male Sprague-Dawley rats under chloral hydrate anesthesia $(0.45 \mathrm{~g} / \mathrm{kg}$, administered intraperitoneally) were immobilized in a stereotactic apparatus frame (Shanghai Ruanlong Science and Technology Development Co., Ltd.). A 1-mm-diameter bur hole was made in the skull (0.7-mm anteriorly and 3-mm laterally to bregma), and a microinjector was inserted into the right striatum for blood injection into the caudate putamen (5-mm deep to bregma). Body temperature was kept at $37^{\circ} \pm 0.5^{\circ} \mathrm{C}$ using a feedback-controlled heating pad. Fresh autologous whole blood was drawn into the microinjector by cutting the rat's tail. After 10 minutes of clotting time, $60 \mu \mathrm{l}$ of semicoagulated autologous blood was infused over 6 minutes with a microinfusion pump (Shanghai Alcott Biotech Co., Ltd.). The injection needle was slowly withdrawn 40 minutes after the injection, and the wound was sutured.

\section{Experimental Schedule and Drug Administration}

This study was divided into 3 parts. Part 1 investigated the dose/effect relationship of lithium on neurological deficits $1,3,5,7,14,21$, and 28 days after ICH. Rats were first subjected to ICH as described above. Forty Sprague-Dawley rats were treated with either lithium (Sigma-Aldrich; 30,60 , or $90 \mathrm{mg} / \mathrm{kg}$ intraperitoneally, dissolved in normal saline, $\mathrm{n}=10$ per group) or saline vehicle (intraperitoneally, $\mathrm{n}=10$ per group) immediately after injury, twice daily for 21 days.

Part 2 examined the therapeutic time window for lithium on neurological deficits $1,3,5,7,14,21$, and 28 days after ICH. Sixty Sprague-Dawley rats were treated with either lithium $(60 \mathrm{mg} / \mathrm{kg}$, intraperitoneally) or saline vehicle, twice daily for 21 days. Animals were divided into the following 6 groups ( $\mathrm{n}=10$ per group) according to the time of delayed treatment after ICH: 1) lithium treatment 2 hours after ICH;2) saline vehicle treatment 2 hours after $\mathrm{ICH}$; 3) lithium treatment 6 hours after ICH; 4) saline vehicle treatment 6 hours after ICH; 5) lithium treatment 10 hours after $\mathrm{ICH}$; and 6) saline vehicle treatment 10 hours after ICH.

In Part 3, lithium $(60 \mathrm{mg} / \mathrm{kg})$ was administered intraperitoneally 2 hours after ICH induction (day 0), and then twice daily for 1 day (for Western blotting), for 3 days (for histological assay), and for 21 days (for hemispheric atrophy measurement). An identical amount of saline was injected intraperitoneally into the ICH-vehicle group, using the same schedule. The dosage $(60 \mathrm{mg} / \mathrm{kg})$ of lithium and timing of administration ( 2 hours after ICH) used were based on the results of Parts 1 and 2. Figure 1A provides details of the experimental schedule.

\section{Assessment of Behavioral Outcomes}

Behavioral testing ( $\mathrm{n}=10$ rats per group) was conducted on Days 1, 3, 5, 7, 14, 21, and 28 using a forelimb placing test and a corner turn test, which were monitored by 2 experimenters who were blind to both neurological and treatment conditions. All behavioral tests were performed the same way as in the study of Hua et al. ${ }^{12}$

\section{Forelimb Placing Test}

Animals were held by their torsos, allowing the forelimb to hang free. Independent testing of each forelimb was induced by brushing the respective vibrissae on the edge of a table top once per trial for 10 trials. Intact animals place the forelimb quickly onto the countertop. Depending on the extent of injury, placing of the forelimb contralateral to the injury may be impaired. A score of 1 was given each time the rat placed its forelimb on the edge of the tabletop in response to the vibrissae stimulation, and the percentage of trials in which the rat placed the appropriate forelimb on the edge of the countertop in response to the vibrissae stimulation was determined.

\section{Corner Turn Test}

The rat was allowed to proceed into a corner, which had an angle of $30^{\circ}$. To exit the corner, the rat could turn either to the left or the right; only turns involving full rearing along either wall were included (i.e., ventral tucks or horizontal turns were excluded). Depending on the extent of injury, rats may show a tendency to turn to the side of the injury. This was repeated 10 to 15 times, with at least 30 seconds between trials, and the percentage of right turns was calculated. The rats were not picked up immediately after each turn so that they did not develop an aversion to their prepotent turning response.

\section{Morphometric Measurement of Hemispheric Atrophy}

Forty-two days after ICH, rats ( $\mathrm{n}=6$ per group) were anesthetized and killed by decapitation so that hemispheric atrophy could be analyzed by an experimenter who was blind to experimental conditions throughout the 
A

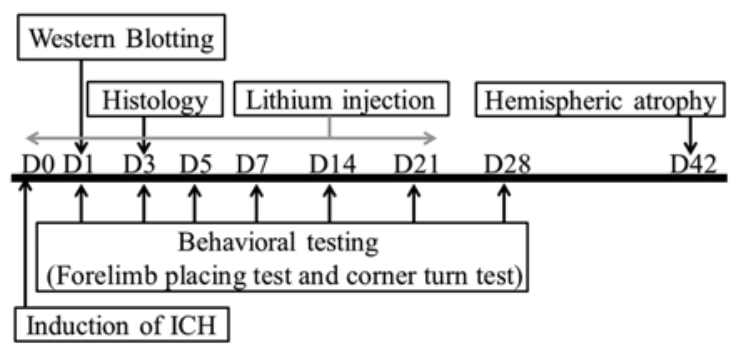

B

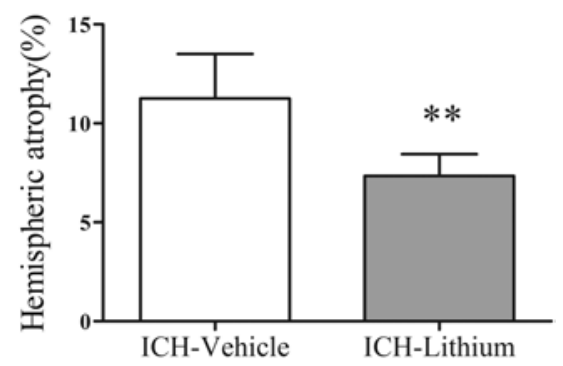

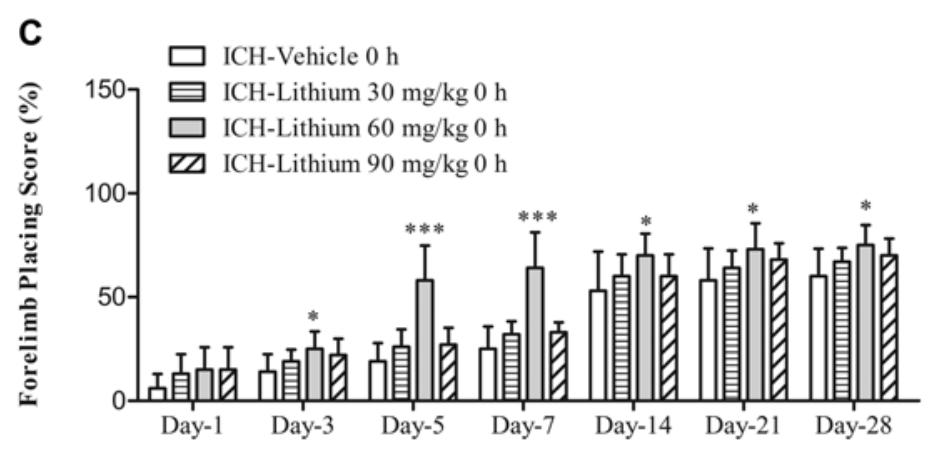

D

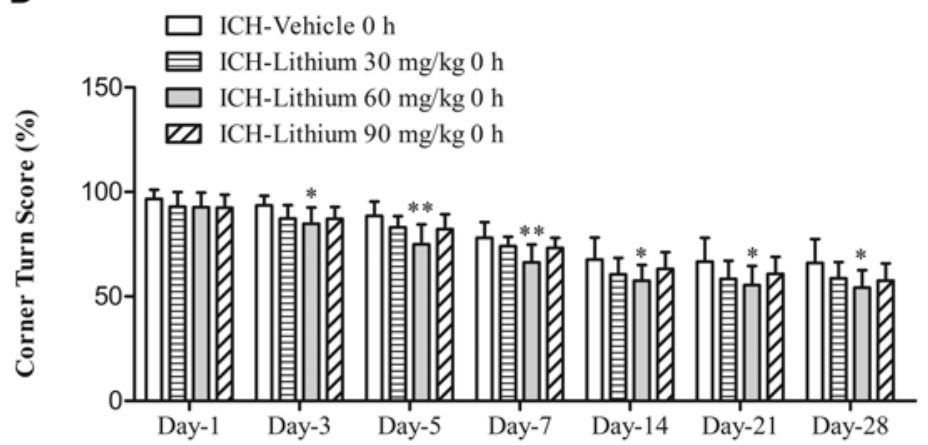

FIG. 1. Schedule of the experiment, dose effect of lithium, and results of hemispheric atrophy. A schedule of the experiment is shown (A). Only lithium-treated groups (60 mg/kg) performed better than did the saline-treated groups 3, 5, 7, 14, 21, and 28 days after $\mathrm{ICH}$ in both the forelimb placing test (C) and the corner turn test (D; $n=10$ rats per group). At 42 days, the lithium-treated group exhibited significantly less hemispheric atrophy than did the saline-treated group ( $\mathbf{B} ; n=6$ rats per group). Bars represent mean \pm SD. ${ }^{*} p<0.05,{ }^{* *} p<0.01,{ }^{* * *} p<0.001$ versus $\mathrm{ICH}$-vehicle.

entire process, as in the study of Kang et al..$^{15}$ Briefly, we immediately removed the brain and 2,3,5-triphenyltetrazolium chloride-stained 5 serial slices (1-mm thick). We calculated total hemispheric volume and then expressed hemispheric atrophy as a percentage of contralateral hemispheric volume.

\section{Histological Assay}

On Day 3 after ICH, rats ( $\mathrm{n}=6$ per group) were reanesthetized and perfused through the heart with $50 \mathrm{ml}$ of cold saline and $50 \mathrm{ml}$ of $4 \%$ paraformaldehyde, in $0.1 \mathrm{~mol} / \mathrm{L}$ of phosphate-buffered saline (PBS). After 24 hours of fixation in $4 \%$ paraformaldehyde, brains were cryoprotected with $30 \%$ sucrose for 24 hours and then cut into $30-\mu \mathrm{m}$ sections using a cryostat (Leica CM 1900), as has been described elsewhere. ${ }^{5,14}$ Immunostaining was then performed using antibodies against myeloperoxidase (MPO, 1:100, Bioss) and OX42 (CD11b, 1:50, AbD Serotec) to evaluate neutrophils and microglia, respectively. Alexa 488-conjugated goat antimouse (1:200, Invitrogen) and Alexa 594-conjugated goat antirabbit (1:200, Invitrogen) were used as the secondary antibodies.

Terminal deoxynucleotidyl transferase-mediated deoxyuridine triphosphate nick-end labeling (TUNEL) staining was conducted for in situ DNA fragmentation detection ( $\mathrm{n}=6$ rats per group), as previously described. ${ }^{8}$ Tissue sections were rinsed with $0.01 \mathrm{~mol} / \mathrm{L}$ PBS and incubated with $0.1 \%$ Triton X-100 on ice for 2 minutes. The sections were then washed and incubated with a TUNEL reaction mixture (1-step TUNEL apoptosis assay kit, Beyotime Institute of Biotechnology) for 60 minutes at $37^{\circ} \mathrm{C}$ in a humidified atmosphere in the dark. The slides were rinsed three times with $0.01 \mathrm{~mol} / \mathrm{L}$ PBS to terminate the reaction. After the slides were washed, the tissue sections were mounted in $50 \%$ glycerol dissolved in $0.01 \mathrm{~mol} / \mathrm{L} \mathrm{PBS}$.

Coronal sections taken through the center of the hem- 
orrhagic lesion were analyzed via the counting of markerspecific cells by an experimenter blind to experimental condition throughout the entire section ( 3 sections per each antibody staining, $1 \mathrm{~mm}$ in width). The total cell counts in these sections were then converted to cell densities, for purposes of quantification and comparison between groups.

\section{Western Blotting}

At 24 hours after ICH induction, rats ( $\mathrm{n}=6$ per group) were killed by decapitation, and the brains were immediately extracted. Ipsilateral hemisphere homogenates were centrifuged at $10,000 \mathrm{~g}$ for 15 minutes, and $50 \mu \mathrm{g}$ of the proteins obtained were separated using $10 \%$ sodium dodecyl sulfate-polyacrylamide gel electrophoresis gels as in the study of Sinn et al. ${ }^{27}$ Blots were transferred to nitrocellulose membranes and incubated in blocking buffer $5 \%$ skimmed milk in Tris-buffered saline with Tween [TBST]: $50 \mathrm{mM}$ Tris at $\mathrm{pH} 7.5,0.15 \mathrm{mM} \mathrm{NaCl}$, and $0.05 \%$ Tween 20), and then with anti-phosphorylated-GSK-3 $\beta$ Ser9 (p-GSK-3ß; 1:500, sc-11757, Santa Cruz Biotechnology), anti-GSK-3 $\beta$ (1:500; sc-81462, Santa Cruz Biotechnology), anti-phosphorylated- $\beta$-catenin Ser33/37/Thr41 (p- $\beta$-catenin, 1:1000; 9561S, Cell Signaling), anti- $\beta$ catenin (1:100; bs-1165R, Bioss), anti-collapsin response mediator protein 2 (CRMP2, 1:100; bs-1790R, Bioss), anti-phosphorylated-CRMP2 Thr514 (p-CRMP2, 1:100; bs-3068R, Bioss), anti-heat shock protein 70 (HSP70, 1:100; bs-0244R, Bioss), anti-cyclooxygenase-2 (COX-2, 1:100; bs-10411R, Bioss), and anti-nuclear factor- $\mathrm{\kappa B}$ (NFкB) p65 (1:100; bs-0465R, Bioss) in TBST at $4^{\circ} \mathrm{C}$ overnight. They were then treated with corresponding secondary antibodies conjugated with horseradish peroxidase in TBST for 1 hour at room temperature. Anti- $\beta$-actin antibody (1:700; TA-09, ZSGB-BIO) was used as a control. Relative optical densities were determined by comparing measured values with the mean values of the ICH-vehicle group. Detections were performed by densitometry using the enhanced chemiluminescence detection system (ECL, Tanon-5200).

\section{Statistical Analysis}

All data were presented as means \pm SDs. GraphPad Prism (version 5.01) was used for all statistical analysis. Because we did not assume that the data showed normal distributions, we used a nonparametric method (the Mann-Whitney U-test) to compare variables between the 2 groups. A p value $<0.05$ was considered a statistically significant difference.

\section{Results}

\section{Lithium and Sensorimotor Outcomes in ICH Rats}

After the injury, immediate treatment of ICH rats with lithium at the dose of $60 \mathrm{mg} / \mathrm{kg}$, but not 30 or $90 \mathrm{mg} / \mathrm{kg}$, significantly improved the outcomes of behavioral tests 3 , $5,7,14,21$, and 28 days after ICH, compared with vehicle (Fig. 1C and D). To optimize the therapeutic time points, lithium $(60 \mathrm{mg} / \mathrm{kg})$ was administered 2,6 , and 10 hours after ICH. It was found that neurological deficits were ameliorated beginning at 3 days after ICH when lithium was administered 2 hours after the insult; this effect was similar to that of lithium administered immediately after ICH. When lithium was administered 6 hours after ICH the ameliorating effect was observed 14 days after the insult. No effect was observed when lithium was administered 10 hours after ICH (Fig. 2). In behavioral tests, we also observed that lithium at the dose of $60 \mathrm{mg} / \mathrm{kg}$, initiated from 2 hours after the injury, produced more significant improvement in the forelimb placing test (on Days 3, 5, 7, 14, 21, and 28; ICH-vehicle: $15.0 \pm 7.1 \%, 20.0 \pm 8.2 \%$, $26.0 \pm 9.7 \%, 51.0 \pm 17.9 \%, 57.0 \pm 14.2 \%$ and $60.0 \pm 13.3 \%$, respectively; ICH-lithium: $24.0 \pm 8.4 \%, 55.0 \pm 12.7 \%, 60.0$ $\pm 14.1 \%, 70.0 \pm 8.2 \%, 72.0 \pm 11.4 \%$, and $72.0 \pm 7.9 \%$, respectively; $<<0.05, \mathrm{p}<0.001, \mathrm{p}<0.001, \mathrm{p}<0.05, \mathrm{p}<0.05$ and $\mathrm{p}<0.05$, respectively, Mann-Whitney U-test; Fig. 2A). For both tests, on Day 1, there were differences in neurological deficits between the groups, but they did not reach statistical significance (Fig. 2A and B).

\section{Lithium and Hemispheric Atrophy}

A hemispheric area analysis conducted 42 days after the ICH revealed significant lesion hemisphere atrophy. The lithium-posttreated group $(7.3 \% \pm 1.1 \%)$ exhibited significantly less hemispheric atrophy than did the salinetreated group $(11.3 \% \pm 2.3 \%, \mathrm{n}=6$ rats per group, $\mathrm{p}<0.01$, Mann-Whitney U-test; Fig. 1B).

\section{Lithium and Number of TUNEL-Positive Cells, MPO-Positive Neutrophils, and OX42-Positive Microglial Cells 3 Days After ICH}

TUNEL staining revealed a high density of positively stained cells in the perihematomal areas 3 days after ICH (Fig. 3A). Lithium significantly reduced the total number of TUNEL-positive cells (ICH-lithium, $91.2 \pm 22.4$ cells/ $\mathrm{mm}^{2}$, vs ICH-vehicle, $269.3 \pm 43.3$ cells $/ \mathrm{mm}^{2}, \mathrm{n}=6$ rats per group; $\mathrm{p}<0.01$, Mann-Whitney U-test). The ICHlithium group also exhibited a significantly lower number of MPO-positive neutrophils (ICH-lithium $123.8 \pm 36.5$ cells $/ \mathrm{mm}^{2}$, ICH-vehicle $219.3 \pm 47.8$ cells $/ \mathrm{mm}^{2}, \mathrm{n}=6$ rats per group; $\mathrm{p}<0.01$, Mann-Whitney U-test), and OX42positive (CD11b-positive ) microglia (ICH-lithium $22.5 \pm$ 5.9 cells $/ \mathrm{mm}^{2}$, ICH-vehicle $115.5 \pm 33.4$ cells $/ \mathrm{mm}^{2}, \mathrm{n}=6$ rats per group; $\mathrm{p}<0.01$, Mann-Whitney U-test) than the ICH-vehicle group at 3 days post-ICH (Fig. 3B).

\section{Lithium Posttreatment and Activity of GSK-3 $\beta$}

Lithium posttreatment upregulated the in vivo $\mathrm{p}-\mathrm{GSK}-$ $3 \beta /$ GSK- $3 \beta$ by 1.5 -fold and downregulated $p-\beta$-catenin $/ \beta$ catenin and $\mathrm{p}$-CRMP2/CRMP2 by 0.6 - and 0.8 -fold, respectively, compared with the ICH-vehicle group at 24 hours after ICH $(n=6$ rats per group; $p<0.01, p<0.01$ and $\mathrm{p}<0.05$, respectively, Mann-Whitney U-test; Fig. 4).

\section{Lithium Posttreatment and Inflammation- and Cell Survival-Related Protein Expression}

Western blotting for NF-кB p65, COX-2, and HSP70 revealed that lithium posttreatment reduced the levels of inflammation-related proteins and increased the levels of cell survival-related protein. The relative optical density of NF-кB p65, COX-2, and HSP70 were 33\% lower, $28 \%$ 

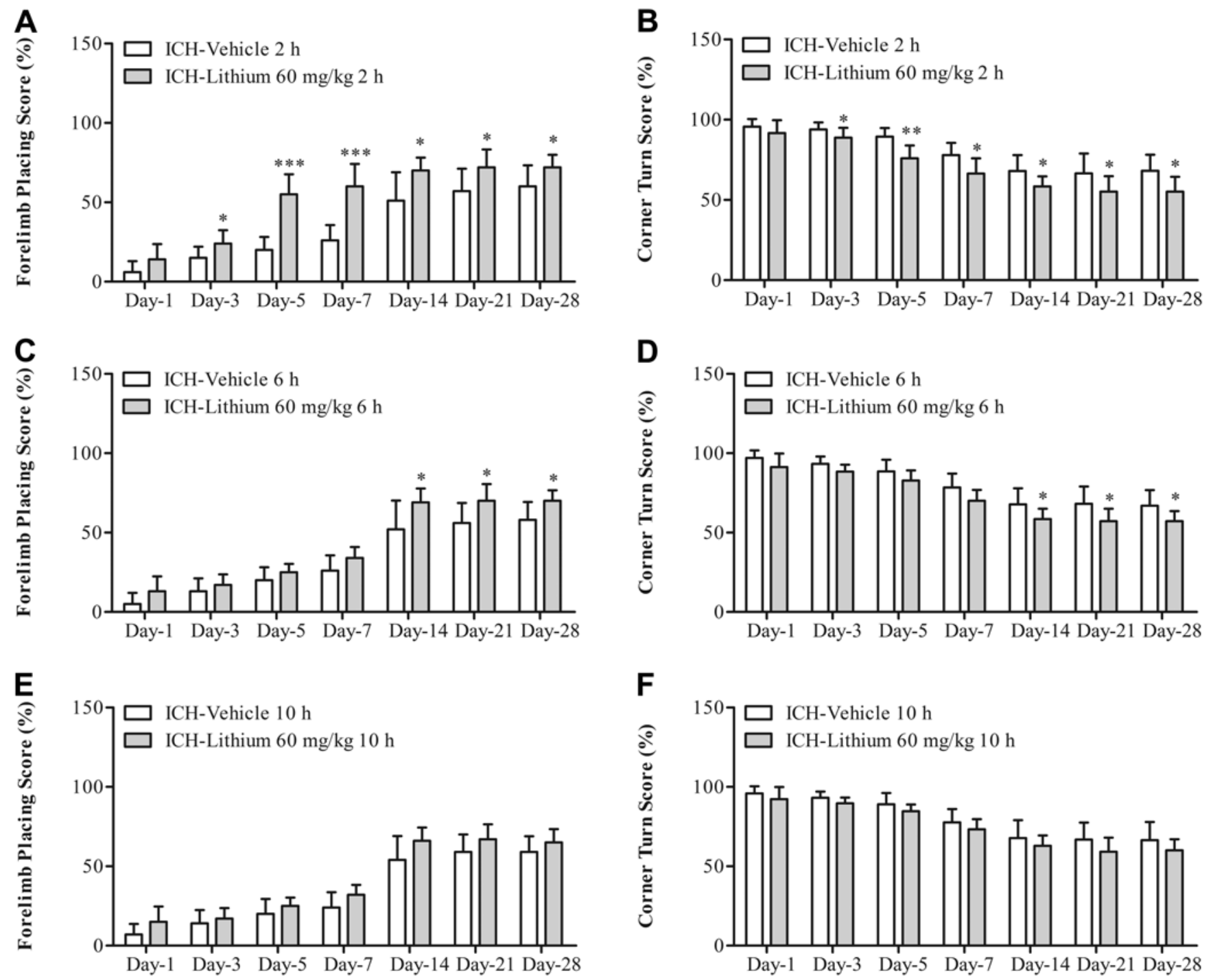

FIG. 2. Therapeutic time window study. Lithium $(60 \mathrm{mg} / \mathrm{kg})$, when administered 2 hours after $\mathrm{ICH}$, ameliorated neurological deficits beginning 3 days after ICH (A and B). These effects were also observed beginning 14 days after ICH when lithium was administered 6 hours $(C$ and $D)$, but not 10 hours $(E$ and $F)$, after the insult. Bars represent mean $\pm S D$. ${ }^{*} p<0.05,{ }^{* *} p<0.01,{ }^{* * *} p<0.001$ versus $\mathrm{ICH}$-vehicle.

lower, and $22 \%$ higher, respectively, in the ICH-lithium group than in the ICH-vehicle group $(n=6$ rats per group; $\mathrm{p}<0.01, \mathrm{p}<0.05$, and $\mathrm{p}<0.05$, respectively, Mann-Whitney U-test; Fig. 5).

\section{Discussion}

In this study, we found that in vivo posttreatment of ICH rats with lithium for 21 days, at a therapeutically relevant concentration of $60 \mathrm{mg} / \mathrm{kg}$ initiated from 2 hours after the injury, significantly attenuated the neurological deficits of ICH, reduced hemispheric atrophy, and exerted neuroprotective effects (anti-inflammation and inhibition of apoptosis) via inactivation of GSK-3 $\beta$.

The first aim of the present study was to investigate whether the lithium posttreatment ameliorated behavioral deficits and brain atrophy after experimental ICH in rats. Hemispheric hemorrhage, affecting the basal ganglia, has been reported to cause debilitating sensorimotor deficits in humans. ${ }^{9,25}$ Intending to achieve a translational perspective, we included sensorimotor assessment in our experiment, consisting of the forelimb placing test and corner turn test. Both the forelimb placing test and the corner turn test have been widely used to evaluate lateralizing behaviors as well as sensorimotor impairments in preclinical ICH studies. ${ }^{12}$ Lithium pretreatment significantly ameliorated neurological deficits at 2 days after ICH via anti-inflammation and reducing brain edema in a rat collagenase ICH model. ${ }^{15}$ The time course of edema is similar in both the collagenase and blood infusion ICH models and gradually resolves over a week. ${ }^{30}$ Any treatment that lessened edema would likely result in significantly better behavioral scores compared with controls experiencing greater swelling, and controls would eventually have similar scores once edema resolved..$^{21}$ The behavioral tests of the lithium pretreatment 


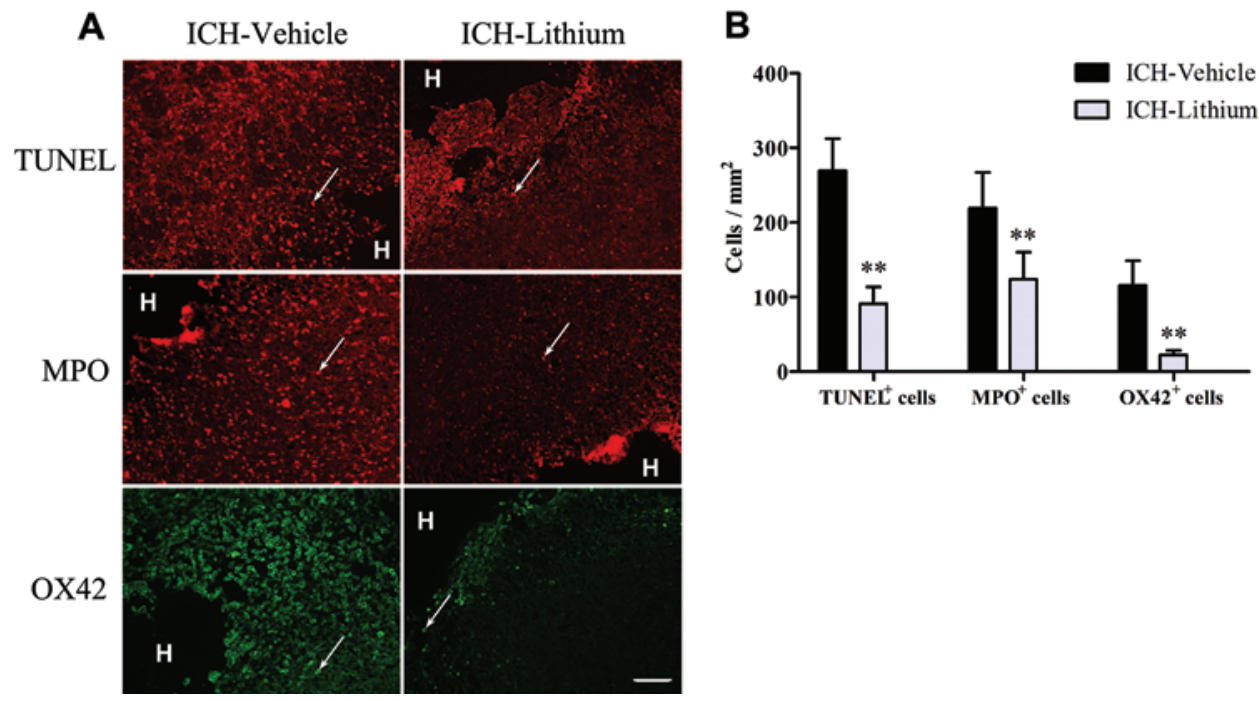

FIG. 3. Histological analysis of apoptosis and inflammation in perihematomal regions. A: Lithium posttreatment for 3 days significantly reduced the numbers of TUNEL-positive cells, MPO-positive neutrophils, and OX42-positive microglia in the perihematomal areas as compared with the $\mathrm{ICH}$-vehicle group. Bar $=50 \mu \mathrm{m} . \mathrm{H}=$ hematoma. Arrow indicates marker-specific cell. TUNEL staining and immunostaining techniques were used; $n=6$ rats per group. B: Quantifications showed significant reductions in apoptosis and inflammation in the ICH-lithium group. Seventy-two representative sections were averaged in all the TUNEL-positive cells, MPO-positive neutrophils, and OX42-positive microglia counts. Bars represent mean \pm SD. ${ }^{* *} p<0.01$ versus ICH-vehicle. Figure is available in color online only.

study were performed only in the acute period (the first 2 days) when edema potentially confounded the interpretation of behavioral data. Although the same investigators evaluated the long-term effects of lithium on the functional recovery after $\mathrm{ICH}$ in another study, they administered lithium both before and after the induction of $\mathrm{ICH} .{ }^{16}$ In fact, pretreatment has little practical clinical meaning, and the effects of posttreatment were unclear based on their study.

In the present study, we performed the behavioral tests for up to 28 days. The time points of behavioral tests selected in the present study were $1,3,5,7,14,21$, and 28 days after $\mathrm{ICH}$, which were consistent with our previous study. ${ }^{20}$ These were crucial time points in a rat model of semi-coagulated autologous blood ICH and allowed continuous monitoring of neurological deficits after $\mathrm{ICH}$, even after resolution of edema. ${ }^{12,20}$ After exploring the therapeutic window both in terms of dose and delayed administration, we found that lithium at the dose of $60 \mathrm{mg} / \mathrm{kg}$ and administered within 2 hours after the injury had the best neuroprotective effect in this study. Therefore, this dosage of lithium $(60 \mathrm{mg} / \mathrm{kg})$ and timing (2 hours after the injury) was used in following studies.
A
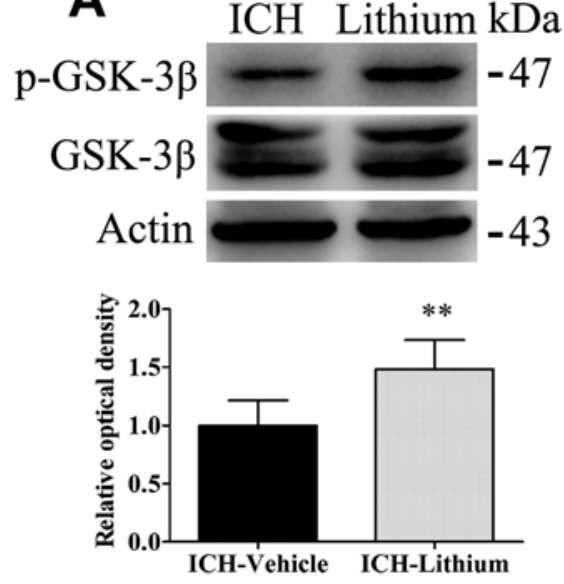

p-GSK-3 $\beta /$ GSK-3 $\beta$
B
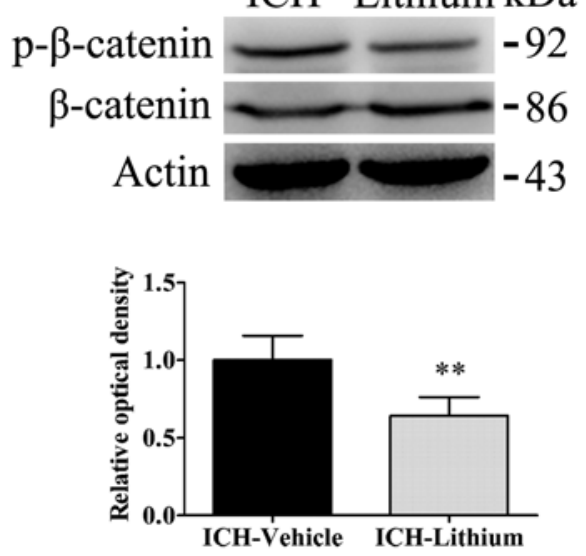

$\mathrm{p}-\beta$-catenin $/ \beta$-catenin
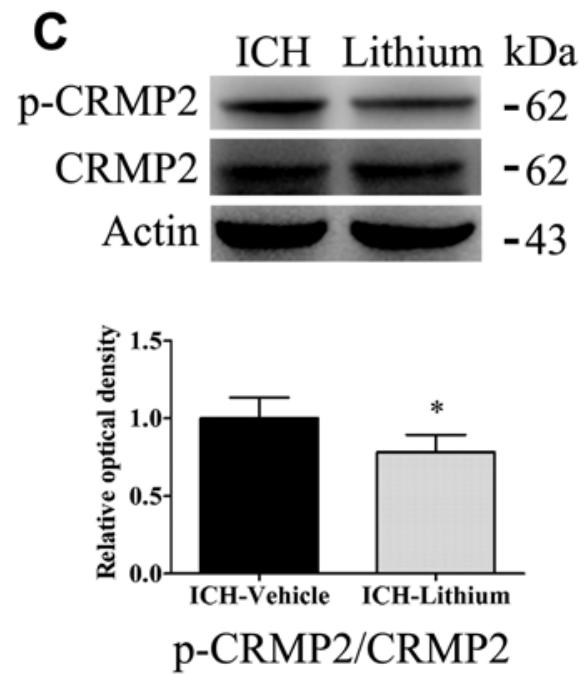

FIG. 4. Representative Western blots (upper row) and densitometric quantification (lower row) of GSK-3 3 . At 24 hours after ICH, lithium posttreatment modulated p-GSK-3 $\beta /$ GSK-3 $\beta$ (A), p- $\beta$-catenin/ $\beta$-catenin (B), and p-CRMP2/CRMP2 (C) expressions by 1.5-, 0.6-, and 0.8-fold, respectively, versus the ICH-vehicle groups. Bars represent mean $\pm S D$. ${ }^{*} p<0.05,{ }^{* *} p<0.01$ versus ICHvehicle; $n=6$ rats per group. 

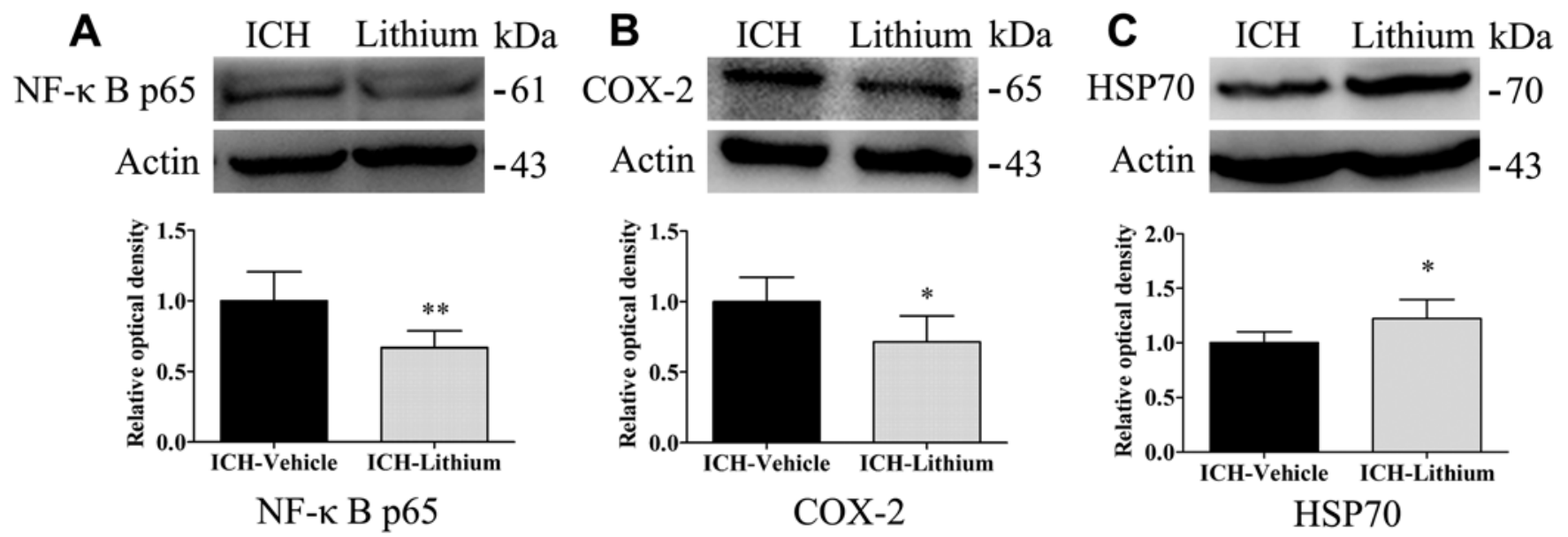

FIG. 5. Representative Western blots (upper row) and densitometric quantification (lower row) of inflammation and cell survivalrelated protein expression. Relative optical density measurements showed that lithium posttreatment reduced NF- $\mathrm{B}$ p 65 (A) and COX-2 (B), and increased HSP70 (C) expressions. Results are representative of 2 experiments. Bars represent mean \pm SD. ${ }^{*} p<$ $0.05,{ }^{* *} p<0.01$ versus $\mathrm{ICH}$-vehicle; $n=6$ rats per group.

Neuroprotective-relevant dose posttreatment of lithium $(60 \mathrm{mg} / \mathrm{kg})$ significantly improved the outcomes of behavioral tests $3,5,7,14,21$, and 28 days after ICH (compared with vehicle). We previously reported that hematoma and brain edema were resolved 14 days after ICH naturally. ${ }^{20}$ In the present study, 14, 21, and 28 days after ICH (edema should be resolved based on other previous studies and our own previous study), lithium posttreatment still improved functional outcomes (compared with vehicle), indicating that the effects of lithium, in addition to anti-inflammation, might be associated with sensorimotor recovery. Although administration of lithium was stopped after 21 days following $\mathrm{ICH}$, its ameliorating effects on neurological deficits could still be observed at 28 days, and reduction of hemispheric atrophy could still be observed at 42 days, indicating that the beneficial effects were long lasting.

Inflammation plays an important role in secondary brain injury after ICH. Inflammation leads to cell apotosis. ${ }^{36}$ Apoptosis represents a prominent form of cell death in the perihematoma regions after ICH. ${ }^{2}$ Microglia, the resident macrophages of the brain, play a critical role as resident immunocompetent and phagocytic cells in the CNS, and become activated within minutes after ICH. ${ }^{18}$ Activated microglia and infiltrating neutrophils release products that induce breakdown of the blood-brain barrier, vasogenic edema, and apoptosis in neurons and glia. ${ }^{26} \mathrm{Af}-$ ter ICH, apoptosis and inflammation peak at 3 days. ${ }^{22,31}$ To evaluate the effects of lithium on apoptotic cells death and inflammation, we examined the numbers of TUNEL-positive cells, MPO-positive neutrophils, and OX42-positive microglia in perihematomal areas 3 days after ICH. We found that all of these cells were reduced in lithium posttreated ICH rats (compared with vehicle). In contrast to the result of this study, a previous study showed that lithium pretreatment did not affect the number of MPO-positive neutrophils 2 days after $\mathrm{ICH} .{ }^{15}$ This discrepancy might be due to the different dosages used, different detection times, and different treatments. The modulation of inflammation and apoptosis by lithium may ameliorate the cell death associated with ICH, producing a reduction in ongo- ing hemispheric atrophy and resulting in overall functional improvement. In the present study, we did find that lithium posttreatment reduced hemispheric atrophy, which might reflect the long-term consequences of lithium on inflammation and cell death. The aforementioned findings support one of our hypotheses that lithium posttreatment ameliorates behavioral and morphological outcomes (brain atrophy) after ICH in rats.

In this study, we additionally intended to show the neuroprotective effect of GSK-3 $\beta$ inhibition. GSK-3 $\beta$ is a serine/threonine kinase, ubiquitously expressed in mammalian eukaryotic cells and one of the main targets in regulation of inflammation and apoptosis..$^{17}$ It is well known that GSK-3 $\beta$ can be phosphorylated at serine residues in which Ser9 phosphorylation renders it inactive. ${ }^{6}$ In this study, we assessed p-GSK-3 $\beta$ (Ser9) /GSK-3 $\beta$ to directly reflect GSK-3 $\beta$ activity. CRMP-2, a microtubuleassociated protein, is a common downstream substrate of GSK-3 $\beta .{ }^{29}$ GSK-3 $\beta$ phosphorylates CRMP-2 at Thr-514 and inactivates it. ${ }^{32}$ GSK-3 $\beta$ also mediates the degradation of $\beta$-catenin molecules by phosphorylating specific amino terminal residues (Ser33, Ser37, and Thr41). ${ }^{13}$ Inhibition of GSK-3 $\beta$ can dephosphorylate CRMP-2 and also lead to activation of cell-survival transcription factors such as $\beta$-catenin, heat-shock factor-1 (HSF-1), cAMP response element-binding protein, and activator protein- $1 ., 7$ To lend further strength to p-GSK-3 $\beta$ assay results, we measured p-CRMP2 (Thr-514) and p- $\beta$-catenin (Ser33, Ser37, and Thr41) levels. Previous studies showed that treatment of lithium results in reduced phosphorylation and subsequent accumulation of $\beta$-catenin, which has been widely used as an indirect assay of GSK-3 $\beta$ activity. ${ }^{11,23,33,34}$

The results of our present study showed that the expression of $p$-GSK-3 $\beta /$ GSK-3 $\beta$ increased and $p-\beta$-catenin/ $\beta$ catenin, p-CRMP2/ CRMP2 decreased (in the ipsilateral hemisphere) in the lithium-posttreated group (compared with vehicle) 1 day after experimental ICH, indicating that the dosage of lithium used in this study was effective in inhibiting GSK-3 $\beta$ activity.

GSK-3 $\beta$ regulates many inflammatory and apoptotic 
molecules. ${ }^{2}$ In this study we investigated NF- $\mathrm{B}, \mathrm{COX}-2$, and HSP70. NF- $\kappa$ B plays a key role in regulating inflammation in brain pathologies. ${ }^{35}$ Moreover, NF- $\kappa \mathrm{B}$ is a key regulator of transcription for a variety of proinflammatory cytokines, chemokines, and inflammatory enzymes (such as COX-2 and the inducible form of NO synthase). ${ }^{35}$ An in vitro study demonstrated that treatment of colorectal cancer with lithium reduced the expression of NF- $\kappa \mathrm{B}$ through inhibiting the activation of GSK-3 $3 .{ }^{19} \mathrm{NF}-\kappa \mathrm{B}$ p65 is a subunit of the NF- $\kappa \mathrm{B}$ transcription complex. The expression of NF- $\kappa B$ p 65 was used to reflect the expression of NF- $\kappa B$ in this study. It has been reported that the inflammatory enzyme COX-2 is induced in endothelial cells and perivascular cells, as well as infiltrating leukocytes 1 day after $\mathrm{ICH} .{ }^{10}$ In the present study, lithium inactivation of GSK$3 \beta$ resulted in decreased expression of NF- $\kappa \mathrm{B}$, and NF- $\kappa \mathrm{B}$ transcriptionally regulated COX-2 (in the ipsilateral hemisphere) in the lithium-posttreated group (compared with vehicle), indicating that inactivation of GSK-3 $\beta$ might be correlated with the reduction of two proinflammatory-associated proteins, NF-אB and COX-2. This might be one of the anti-inflammation mechanisms in lithium-posttreated $\mathrm{ICH}$ rats.

Inhibition of GSK-3 $\beta$ also contributes to the activation of HSF-1 (a transcription factor), and HSF-1 regulates the expression of cytoprotective protein HSP70.${ }^{4} \mathrm{HSP} 70$ exerts a wide variety of neuroprotective effects against apoptosis. $^{28}$ These occur by antagonizing apoptosis-inducing factors, preventing mitochondrial cytochrome $\mathrm{c}$ release and caspase activation, and suppressing c-Jun N-terminal kinase activation. ${ }^{4}$ Our study found that lithium inactivation of GSK-3 $\beta$ resulted in increased expression of HSP70 (in the ipsilateral hemisphere) in the lithium-posttreated group (compared with vehicle), indicating that inactivation of GSK-3 $\beta$ might be correlated with increased HSP70, associated with cell survival, which may be one of the mechanisms of inhibition of apoptosis in lithium-posttreated ICH rats. Added together, these findings support another hypothesis of ours, suggesting that lithium posttreatment, through inhibition of GSK-3 $\beta$, alleviates inflammation and cell apoptosis after ICH in rats.

\section{Conclusions}

The present study found that lithium posttreatment $(60$ $\mathrm{mg} / \mathrm{kg}$, initiated from 2 hours after injury) has attenuated neurological deficits, reduced hemispheric atrophy, and inhibited inflammation and apoptosis in a rat model of semicoagulated autologous blood ICH through inactivation of GSK-3 $\beta$. In addition, we found that the effect of earlier administration of lithium (initiated from 2 hours after injury) is much better than that of later treatment (initiated from 6 hours after injury). However, further in vitro studies are needed to verify the in vivo results of the current study and to investigate the activity of NF- $\mathrm{-B}$ and HSF-1, which play important roles in regulating inflammation and HSP70 expression.

\section{Acknowledgments}

This work was supported by funding from the National Natural Science Foundation of China (grant no. 81271287, to Dr. X. Tang) and the Graduate Innovative Research Project of Harbin Medical University (grant no. YJSCX2015- 49HYD, to Dr. Zheng).

\section{References}

1. Aronowski J, Hall CE: New horizons for primary intracerebral hemorrhage treatment: experience from preclinical studies. Neurol Res 27:268-279, 2005

2. Beurel E, Grieco SF, Jope RS: Glycogen synthase kinase-3 (GSK3): regulation, actions, and diseases. Pharmacol Ther 148:114-131, 2015

3. Bijur GN, Jope RS: Opposing actions of phosphatidylinositol 3 -kinase and glycogen synthase kinase- $3 \beta$ in the regulation of HSF-1 activity. J Neurochem 75:2401-2408, 2000

4. Chiu CT, Chuang DM: Molecular actions and therapeutic potential of lithium in preclinical and clinical studies of CNS disorders. Pharmacol Ther 128:281-304, 2010

5. Chu K, Jeong SW, Jung KH, Han SY, Lee ST, Kim M, et al: Celecoxib induces functional recovery after intracerebral hemorrhage with reduction of brain edema and perihematomal cell death. J Cereb Blood Flow Metab 24:926-933, 2004

6. Chuang DM, Wang Z, Chiu CT: GSK-3 as a target for lithium-induced neuroprotection against excitotoxicity in neuronal cultures and animal models of ischemic stroke. Front Mol Neurosci 4:15, 2011

7. Fang WQ, Ip JPK, Li R, Ng YP, Lin SC, Chen Y, et al: Cdk5mediated phosphorylation of Axin directs axon formation during cerebral cortex development. J Neurosci 31:1361313624,2011

8. Gavrieli Y, Sherman Y, Ben-Sasson SA: Identification of programmed cell death in situ via specific labeling of nuclear DNA fragmentation. J Cell Biol 119:493-501, 1992

9. Gebel JM, Broderick JP: Intracerebral hemorrhage. Neurol Clin 18:419-438, 2000

10. Gong C, Ennis SR, Hoff JT, Keep RF: Inducible cyclooxygenase-2 expression after experimental intracerebral hemorrhage. Brain Res 901:38-46, 2001

11. Hedgepeth CM, Conrad LJ, Zhang J, Huang H-C, Lee VM, Klein PS: Activation of the Wnt signaling pathway: a molecular mechanism for lithium action. Dev Biol 185:82-91, 1997

12. Hua Y, Schallert T, Keep RF, Wu J, Hoff JT, Xi G: Behavioral tests after intracerebral hemorrhage in the rat. Stroke 33:2478-2484, 2002

13. Jope RS, Johnson GV: The glamour and gloom of glycogen synthase kinase-3. Trends Biochem Sci 29:95-102, 2004

14. Jung KH, Chu K, Jeong SW, Han SY, Lee ST, Kim JY, et al: HMG-CoA reductase inhibitor, atorvastatin, promotes sensorimotor recovery, suppressing acute inflammatory reaction after experimental intracerebral hemorrhage. Stroke 35:1744-1749, 2004

15. Kang K, Kim YJ, Kim YH, Roh JN, Nam JM, Kim PY, et al: Lithium pretreatment reduces brain injury after intracerebral hemorrhage in rats. Neurol Res 34:447-454, 2012

16. Kang K, Kim YJ, Lee SH, Yoon BW: Lithium fails to enhance neurogenesis in subventricular zone and dentate subgranular zone after intracerebral hemorrhage in rats. Neurol Res 36:79-85, 2014

17. King MK, Pardo M, Cheng Y, Downey K, Jope RS, Beurel E: Glycogen synthase kinase-3 inhibitors: rescuers of cognitive impairments. Pharmacol Ther 141:1-12, 2014

18. Kreutzberg GW: Microglia: a sensor for pathological events in the CNS. Trends Neurosci 19:312-318, 1996

19. Li H, Huang K, Liu X, Liu J, Lu X, Tao K, et al: Lithium chloride suppresses colorectal cancer cell survival and pro-

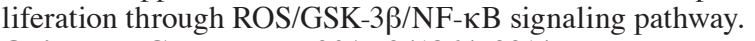
Oxid Med Cell Longev 2014:241864, 2014

20. Liu L, Wang S, Xu R, Zheng J, Tang J, Tang X, et al: Experimental intracerebral haemorrhage: description of a semi-coagulated autologous blood model in rats. Neurol Res 37:874-879, 2015

21. MacLellan CL, Paquette R, Colbourne F: A critical appraisal 
of experimental intracerebral hemorrhage research. J Cereb Blood Flow Metab 32:612-627, 2012

22. Matsushita K, Meng W, Wang X, Asahi M, Asahi K, Moskowitz MA, et al: Evidence for apoptosis after intercerebral hemorrhage in rat striatum. J Cereb Blood Flow Metab 20:396-404, 2000

23. Miller JR, Moon RT: Signal transduction through $\beta$-catenin and specification of cell fate during embryogenesis. Genes Dev 10:2527-2539, 1996

24. Nassar A, Azab AN: Effects of lithium on inflammation. ACS Chem Neurosci 5:451-458, 2014

25. Nys GM, van Zandvoort MJ, de Kort PL, Jansen BP, de Haan EH, Kappelle LJ: Cognitive disorders in acute stroke: prevalence and clinical determinants. Cerebrovasc Dis 23:408416, 2007

26. Qureshi AI, Mendelow AD, Hanley DF: Intracerebral haemorrhage. Lancet 373:1632-1644, 2009

27. Sinn DI, Kim SJ, Chu K, Jung KH, Lee ST, Song EC, et al: Valproic acid-mediated neuroprotection in intracerebral hemorrhage via histone deacetylase inhibition and transcriptional activation. Neurobiol Dis 26:464-472, 2007

28. Takayama S, Reed JC, Homma S: Heat-shock proteins as regulators of apoptosis. Oncogene 22:9041-9047, 2003

29. Wang T, Wu X, Yin C, Klebe D, Zhang JH, Qin X: CRMP-2 is involved in axon growth inhibition induced by RGMa in vitro and in vivo. Mol Neurobiol 47:903-913, 2013

30. Xi G, Fewel ME, Hua Y, Thompson BG Jr, Hoff JT, Keep RF: Intracerebral hemorrhage: pathophysiology and therapy. Neurocrit Care 1:5-18, 2004

31. Xue M, Del Bigio MR: Intracerebral injection of autologous whole blood in rats: time course of inflammation and cell death. Neurosci Lett 283:230-232, 2000

32. Yoshimura T, Kawano Y, Arimura N, Kawabata S, Kikuchi A, Kaibuchi K: GSK-3 $\beta$ regulates phosphorylation of CRMP2 and neuronal polarity. Cell 120:137-149, 2005

33. Yost C, Farr GH III, Pierce SB, Ferkey DM, Chen MM, Kimelman D: GBP, an inhibitor of GSK-3, is implicated in
Xenopus development and oncogenesis. Cell 93:1031-1041, 1998

34. Yost C, Torres M, Miller JR, Huang E, Kimelman D, Moon RT: The axis-inducing activity, stability, and subcellular distribution of beta-catenin is regulated in Xenopus embryos by glycogen synthase kinase 3. Genes Dev 10:1443-1454, 1996

35. Zhao X, Zhang Y, Strong R, Zhang J, Grotta JC, Aronowski $\mathrm{J}$ : Distinct patterns of intracerebral hemorrhage-induced alterations in NF- $\mathrm{KB}$ subunit, iNOS, and COX-2 expression. J Neurochem 101:652-663, 2007

36. Zhou Y, Wang Y, Wang J, Stetler RA, Yang QW: Inflammation in intracerebral hemorrhage: from mechanisms to clinical translation. Prog Neurobiol 115:25-44, 2014

\section{Disclosures}

The authors report no conflict of interest concerning the materials or methods used in this study or the findings specified in this paper.

\section{Author Contributions}

Conception and design: Zheng. Acquisition of data: Liu, Li, J Tang, Zhang. Analysis and interpretation of data: Liu, Li, J Tang, Zhang. Drafting the article: Zheng. Critically revising the article: X Tang. Reviewed submitted version of manuscript: X Tang. Approved the final version of the manuscript on behalf of all authors: X Tang. Administrative/technical/material support: Zheng, Liu, J Tang, Zhang. Study supervision: Zheng.

\section{Correspondence}

Xiaobo Tang, Department of Biopharmaceutical Sciences, College of Pharmacy, Harbin Medical University, 157 Baojian Rd., PO Box 19, Nangang District, Harbin, Heilongjiang 150081, China.email: ty6163@aliyun.com. 